IN liquid crystals long molecules are aligned parallel to one another but are disordered and free to move in the plane perpendicular to their long axis. The ends of the molecules may all be in register (smectic) or not (nematic), with all the chains parallel, or in register with successive layers of molecules tilted in different directions (cholesteric). It has always seemed likely that some synthetic polymers might form liquid crystals but until recently there has been no example of this in a pure polymer. Liquid crystal formers have a characteristic melting behaviour. On heating there is a change from crystal to liquid crystal with a large heat of transition followed a few degrees later by a transition with a small heat to the liquid. One should also be able to observe the partial ordering by $\mathrm{X}$-ray diffraction and the molecular motion in the liquid crystalline phase by NMR.

Beatty and co-workers at Xerox (Macromolecules, 8, 547; 1975) have now described an almost perfect example of such melting behaviour in polydiethylsiloxane. Calorimetry and X-ray diffraction provide supporting evidence and restricted molecular motion was observed by NMR and dielectric measurements. In other respects polydiethylsiloxane is a normally behaved semi-crystalline polymer and the amorphous regions of the structure seem unaffected by the transition in the crystalline parts. Polysiloxane chains are quite flexible although it would seem more reasonable to expect liquid crystalline phases in stiff chains, where the interchain bonding could loosen at lower temperatures than those at which the chains could become free to rotate internally.

The morphology of the liquid crystalline phase and the mechanism of the transition from liquid to liquid

\section{Polymeric liquid crystals}

from Paul Calvert

crystal and from liquid crystal to crystal in terms of chain folding and spherulite formation have yet to be elucidated. The work of Price and Stein (J. Phys. Chem., 77, 396, 399, $409 ; 1973)$ on the crystallisation kinetics of cholesteric liquid crystals provides a starting point for considering what is expected in the polymeric system.

It is possible that other polymer liquid crystalline phases have been overlooked because of their narrow temperature range. Beatty mentions a series of atactic stiff chain acrylates which have similar X-ray diffraction and melting behaviour to liquid crystals. These materials, however, do not form true crystals and probably fit better into the grey area between ordered glasses and disordered crystals. A better example is the phase observed to form at high pressures in polyethylene by Bassett (J. appl. Phys., 45, 4146; 1974). This shows the same behaviour as polydiethylsiloxane though Bassett favours a disordered hexagonal crystal phase at present. He suggests that this phase is the cause of extended chain (large) crystal formation in polyethylene at high pressures. If this is a liquid crystal phase one could expect that extended chain crystals will also grow from polydiethylsiloxane.

There are some examples of solutions of polymers which form liquid crystals. Stiff chain aromatic polyamides in solution in acids form liquid crystals at about $10 \%$ polymer. Poly- $\gamma$-benzyl-L-glutamate in dichloromethane is similar (Duke and DuPré, Macromolecules, 7, 374; 1974; Hines and Samutski, Macromolecules, 6, 793 ; 1973), on the face of it, however, these solution systems are a different case from pure polymer.

This would be a good time for polymer physicists to predict the arrangement of chains in the liquid crystalline phase and to predict which other polymers should form liquid crystals. Current theories of polymer crystallisation seem rather ad hoc and a successful prediction could add respectability. been opened towards the prokaryotes (Wallace and Morowitz, Chromosoma, 40, 121; 1973).

The situation reported for $M$. maculatus was not, however, observed in other organisms (see for example Chilton and McCarthy, Genetics, 74, 605; 1973). Furthermore, the $M$. maculatus satellite had most unusual properties: not only did it contain highly-repetitive DNA, as do all DNA satellites, but most of it consisted of DNA sequences which, as far as the satellite was concerned, were unique. This was, so to speak, a unique observation.

From the same group in Norwich, but with different authorship, there came recently a confirmation of the correlation between B chromosome content and the occurrence of satellite in M. maculatus (Wilmore and Brown, Chromosoma, 51, 337; 1975). This study pointed up a most interesting coincidence: the buoyant density of the $M$. maculatus satellite is identical to that of Paramecium aurelia DNA, to the third decimal place. It also has much the same analytical complexity (compare Gibson and Hewitt, Chromosoma, 38, 121; 1972; and Cummings, Chromosoma, 53, 192; 1975).
Yet more recently, Dover and Henderson (Nature, 259, 57; 1975) have reopened the issue by examining some of the same populations of $M$. maculatus previously examined by Gibson and Hewitt. They did indeed find $\mathbf{B}$ chromosomes, but found no satellite, even in the presence of actinomycin D. In fact, grasshoppers as a group seem to be unusually free of satellites (Wilmore and Brown, op. cit., 1975).

The unusual properties of the satellite observed by Gibson and Hewitt offer a conceivable solution to the problem. It shows the characteristics of an entire genome, rather than those common to other eukaryotic satellites, and furthermore, it is uncannily like a ciliate genome. It seems possible, then, that the DNA of some grasshoppers is contaminated by that of a ciliate, perhaps a ciliate parasite. It is not by any means inconceivable that the distribution of such a parasite would correlate, perhaps loosely, with that of B chromosomes.

Whatever the eventual explanation it would seem that B chromosomes may soon return securely to their former place as potential progenitors of genetic diversity in eukaryotes. Perhaps this is one instance in which a negative finding could ultimately outweigh a positive one.

\section{The early Earth}

from Lynn Margulis

The second College Park Symposium-Chemical Evolution in the Precambrian-was held at the University of Maryland on October 29. November 1. It was sponsored by NASA and the NSF and organised and chaired by Professor Cyril Ponnamperuma, Laboratory of Chemical Evolution, Department of Chemistry, University of Maryland. The proceedings will be published by Academic Press, New York, jointly with last year's College Park Symposium on the giant planets.

WHAT were conditions like 4 thousand million years ago? Despite enormous diversity in training, formal back- 